\title{
Polynomial Interpolation to Boundary Data on Triangles
}

\author{
By R. E. Barnhill* and J. A. Gregory
}

\begin{abstract}
Boolean sum interpolation theory is used to derive a polynomial interpolant which interpolates a function $F \in C^{N}(\bar{T})$, and its derivatives of order $N$ and less, on the boundary $\partial T$ of a triangle $T$. A triangle with one curved side is also considered.
\end{abstract}

1. Introduction. Boolean sum interpolation theory** was first used on triangles by Barnhill, Birkhoff, and Gordon [1] to derive rational functions interpolating the boundary data. The general theory of Boolean sum interpolation is briefly discussed in this paper and a polynomial Boolean sum interpolant is presented, which, for any positive integer $N$, interpolates a function $F \in C^{N}(\bar{T})$, and its derivatives of order $N$ and less, on the boundary $\partial T$ of a triangle $T$. The case $N=0$ corresponds to an interpolant constructed by other means by Nielson [6]. The interpolant requires that certain derivatives of $F$ be compatible at the vertices of $T$, but these conditions can be removed by adding suitable rational terms. The theory is generalized for a triangle with one curved side.

The interpolant can be used to define a piecewise function which is $C^{N}(\Omega)$ over a triangular subdivision of a polygonal region $\Omega$. This has applications to computer aided geometric design and finite element analysis. Finite dimensional, piecewise defined, $C^{N}(\Omega)$ interpolants can be derived by taking the boundary data to be functions interpolating discrete data along the sides. Alternatively, the blending function can be incorporated with finite elements so as to match exactly a given boundary function on $\Omega$; see, for example, Marshall and Mitchell [5], who interpolate over a polygonal region $\Omega$. The general theory of interpolation to boundary data for a triangle with one curved side, presented in Section 5, permits essential boundary conditions to be satisfied exactly.

2. Boolean Sum Interpolation Theory. This section considers conditions which are sufficient for the application of Boolean sum interpolation theory. These conditions motivate the formulation of the projectors considered in Section 3. The interpolation

Received March 15, 1974.

AMS (MOS) subject classifications (1970). Primary 65D05.

Key words and phrases. Bivariate interpolation, Coons patches for triangles, polynomial blending functions, blending function interpolation methods, Boolean sum interpolation, curved boundary finite elements.

* The research of R. E. Barnhill was supported by The National Science Foundation with Grant GP 20293 to the University of Utah, by the Science Research Council with Grants B/SR/9652 at Brunel University and $B / R G / 61876$ at Dundee University, by a N.A.T.O. Senior Fellowship in Science, and by the University of Utah Research Committee.

** The expression "Boolean sum interpolation" is used in this paper, instead of "blending function interpolation", to emphasize the interpolation properties of the projectors per se in the Boolean sums. 
of the function $F$ is first discussed, and this is then generalized to the interpolation of the function $F$ and its derivatives.

THEOREM 2.1. Let $\Gamma_{1}$ and $\Gamma_{2}$ be two subsets of $R^{n}$, and let $F$ be a function defined on $\Gamma_{1} \cup \Gamma_{2}$. Let $P_{1}$ and $P_{2}$ be two interpolation projectors such that $P_{i} F=F$ on $\Gamma_{i}$, and $P_{i} F$ is defined on $\Gamma_{1} \cup \Gamma_{2}, i=1,2$. Then the Boolean sum function,

$$
\left(P_{1} \oplus P_{2}\right) F=\left(P_{1}+P_{2}-P_{1} P_{2}\right) F,
$$

(i) interpolates $F$ on $\Gamma_{1}$,

(ii) interpolates $F$ on $\Gamma_{2}-\Gamma_{1}$ if $P_{1} F$ on $\Gamma_{2}-\Gamma_{1}$ is a linear combination of function evaluations on $\Gamma_{2}$.

Proof. (i) Since $I-P_{1}$ is null on $\Gamma_{1}$, where $I$ is the identity operator, it follows that

$$
F-\left(P_{1} \oplus P_{2}\right) F \equiv\left(I-P_{1}\right)\left(I-P_{2}\right) F
$$

is zero on $\Gamma_{1}$.

(ii) Also, since $\left(I-P_{2}\right) F=0$ on $\Gamma_{2}$,

$$
F-\left(P_{1} \oplus P_{2}\right) F \equiv\left(I-P_{2}\right) F-P_{1}\left(I-P_{2}\right) F
$$

is zero on $\Gamma_{2}-\Gamma_{1}$ if $P_{1}$ on $\Gamma_{2}-\Gamma_{1}$ is a linear combination of function evaluations on $\Gamma_{2}$. Q.E.D.

In practice, $P_{i} F$ usually involves $F$ only on $\Gamma_{i}$. The hypothesis of Theorem 2.1 then becomes that $P_{1} F$ on $\Gamma_{2}-\Gamma_{1}$ is a linear combination of function evaluations on $\Gamma_{1} \cap \Gamma_{2}$, where it is a necessary condition that $\Gamma_{1} \cap \Gamma_{2}$ is not null.

Remark. If the dual hypothesis holds for $\left(P_{2} \oplus P_{1}\right) F$, that is, $P_{2} F$ on $\Gamma_{1}-\Gamma_{2}$ is a linear combination of function evaluations on $\Gamma_{1}$, then

$$
\left(P_{1} \oplus P_{2}\right) F=\left(P_{2} \oplus P_{1}\right) F \quad \text { on } \Gamma_{1} \cup \Gamma_{2}
$$

and hence

$$
P_{1} P_{2} F=P_{2} P_{1} F \quad \text { on } \Gamma_{1} \cup \Gamma_{2} \text {. }
$$

We thus have sufficient conditions that the projectors satisfy the definition of weak commutativity of Gordon and Wixom [4].

The generalization of Theorem 2.1 to the interpolation of function and derivatives on $\Gamma_{1} \cup \Gamma_{2}$ is the following:

THEOREM 2.2. Let $P_{1}$ and $P_{2}$ be two interpolation projectors such that $D^{\alpha} P_{i} F$ $=D^{\alpha} F$ on $\Gamma_{i}$ and $D^{\alpha} P_{i} F$ is defined on $\Gamma_{1} \cup \Gamma_{2}, i=1,2$, for all $|\alpha| \leqslant N$, where

$$
\alpha=\left(\alpha_{1}, \ldots, \alpha_{n}\right) \text { and } D^{\alpha}=\frac{\partial^{|\alpha|}}{\partial x_{1}^{\alpha} 1 \ldots \partial x_{n}^{\alpha}} .
$$

Then

(i) $D^{\alpha}\left(P_{1} \oplus P_{2}\right) F=D^{\alpha} F$ on $\Gamma_{1}$ for all $|\alpha| \leqslant N$,

(ii) $D^{\alpha}\left(P_{1} \oplus P_{2}\right) F=D^{\alpha} F$ on $\Gamma_{2}-\Gamma_{1}$ for all $|\alpha| \leqslant N$ if $D^{\alpha} P_{1} F$ on $\Gamma_{2}-\Gamma_{1}$ is a linear combination of function and derivative evaluations on $\Gamma_{2}$ which are interpolated by $P_{2} F$. 
Proof. The proof is an extension of the proof of Theorem 2.1. The only complication is on $\Gamma_{2}-\Gamma_{1}$ where

$$
D^{\alpha} F-D^{\alpha}\left(P_{1} \oplus P_{2}\right) F \equiv D^{\alpha}\left(I-P_{2}\right) F-D^{\alpha} P_{1}\left(I-P_{2}\right) F
$$

is zero for all $|\alpha| \leqslant N$ if and only if $D^{\alpha} P_{1}\left(I-P_{2}\right) F=0$. A sufficient condition for this to hold is that $D^{\alpha} P_{1}$ on $\Gamma_{2}-\Gamma_{1}$ is a linear combination of function and derivative evaluations on $\Gamma_{2}$. For $|\alpha| \geqslant 1$ some of these derivatives may be of order greater than $N$ and thus we require that these be interpolated by $P_{2}$. Q.E.D.

Note. Since $P_{2} F$ interpolates $D^{\alpha} F$ on $\Gamma_{2}$ for all $|\alpha| \leqslant N$, then, assuming its existence, $\partial^{\beta} / \partial s^{\beta}\left(D^{\alpha} F\right)$ is also interpolated on $\Gamma_{2}$, where $\partial / \partial s$ is any derivative along the set $\Gamma_{2}$. Such derivatives, assuming any necessary compatibility to allow change of order of differentiation, frequently include those required by Theorem 2.2.

Example of Rational Interpolation on Triangles. Consider the standard triangle $T$ with vertices at $V_{1}=(0,1), V_{2}=(1,0)$, and $V_{3}=(0,0)$, where the side opposite the vertex $V_{k}$ is denoted by $E_{k}$. Rational Hermite projectors on $T$ are defined by

$$
\begin{aligned}
T_{1} F= & \sum_{i \leqslant N} \varphi_{i}\left(\frac{x}{1-y}\right)(1-y)^{i} F_{i, 0}(0, y)+\sum_{i \leqslant N} \psi_{i}\left(\frac{x}{1-y}\right)(1-y)^{i} F_{i, 0}(1-y, y) \\
T_{2} F= & \sum_{i \leqslant N} \varphi_{i}\left(\frac{y}{1-x}\right)(1-x)^{i} F_{0, i}(x, 0)+\sum_{i \leqslant N} \psi_{i}\left(\frac{y}{1-x}\right)(1-x)^{i} F_{0, i}(x, 1-x), \\
T_{3} F= & \sum_{i \leqslant N} \varphi_{i}\left(\frac{x}{x+y}\right)(x+y)^{i}\left(\left[\frac{\partial}{\partial x}-\frac{\partial}{\partial y}\right]^{i} F\right)(0, x+y) \\
& +\sum_{i \leqslant N} \psi_{i}\left(\frac{x}{x+y}\right)(x+y)^{i}\left(\left[\frac{\partial}{\partial x}-\frac{\partial}{\partial y}\right]^{i} F\right)(x+y, 0)
\end{aligned}
$$

where the $\varphi_{i}(t)$ and $\psi_{i}(t)=(-1)^{i} \varphi_{i}(1-t)$ are the cardinal basis functions for Hermite two point Taylor interpolation on the interval $[0,1]$. Boolean sum interpolation using these projectors was first considered by Barnhill, Birkhoff, and Gordon [1]. Application of Theorem 2.2 gives the following theorem.

THEOREM 2.3. The Boolean sum functions, $\left(T_{i} \oplus T_{j}\right) F=\left(T_{i}+T_{j}-T_{i} T_{j}\right) F$, $i \neq j ; i, j=1,2,3$, interpolate $F \in C^{N}(\bar{T})$ and its derivatives of order $N$ and less on $\partial T$, provided that $F$ satisfies the compatibility conditions

$$
\left(\frac{\partial^{m+n} F}{\partial s_{i}^{m} \partial s_{j}^{n}}\right)\left(V_{k}\right)=\left(\frac{\partial^{n+m} F}{\partial s_{j}^{n} \partial s_{i}^{m}}\right)\left(V_{k}\right), \quad m, n \leqslant N ; m+n>N,
$$

where $V_{k}$ is the vertex with adjacent sides $E_{i}$ and $E_{j}$, and $\partial / \partial s_{l}$ denotes differentation along the side $E_{l}$.

Proof. By affine transformation and symmetry, it is sufficient to consider the case $\left(T_{1} \oplus T_{2}\right) F . T_{1} F$ and $T_{2} F$ interpolate on $\Gamma_{1}=E_{2} \cup E_{3}$ and $\Gamma_{2}=E_{1} \cup E_{3}$, respectively. With reference to the hypotheses of Theorem $2.2, D^{\alpha} T_{1} F,|\alpha| \leqslant N$, on $\Gamma_{2}-\Gamma_{1}=E_{2}$ involves linear combinations of 


$$
\left(\frac{\partial^{n+m} F}{\partial y^{n} \partial x^{m}}\right)(0,0) \text { and }\left(\left[-\frac{\partial}{\partial x}+\frac{\partial}{\partial y}\right]^{n} \frac{\partial^{m} F}{\partial x^{m}}\right)(1,0), \quad 0 \leqslant m, n \leqslant N .
$$

The latter derivative is interpolated by $T_{2} F$, since tangential derivatives along the side are automatically interpolated. Also, $T_{2} F$ interpolates $F_{0, n}(x, 0)$ and hence interpolates

$$
\left(\frac{\partial^{m+n} F}{\partial x^{m} \partial y^{n}}\right)(0,0), \quad 0 \leqslant m, n \leqslant N .
$$

Thus the hypotheses of Theorem 2.2 are satisfied if $F \in C^{N}(\bar{T})$ and satisfies the compatibility conditions $(2.5)$ at the vertex $V_{3}=(0,0)$. Q.E.D.

Precision. The precision set is the set of polynomials for which the interpolant is exact and is important in that it indicates the order of accuracy of the interpolant. The precision set of the Boolean sum operator $P_{1} \oplus P_{2}$ is at least that of $P_{2}$ since

$$
I-\left(P_{1} \oplus P_{2}\right) \equiv I-P_{2}-P_{1}\left(I-P_{2}\right)
$$

and $I-P_{2}$ is null on the precision set of $P_{2}$. Thus the Boolean sum operator $P_{1} \oplus P_{2}$ has at least the interpolation properties of the projector $P_{1}$ and the precision set of the projector $P_{2}$.

3. Polynomial Interpolation on Triangles. By affine invariance it is sufficient to consider the standard triangle $T$ defined above. Projectors $P_{1}$ and $P_{2}$ are considered, which satisfy the conditions of Theorem 2.2 and which respectively interpolate $F \in$ $C^{N}(\bar{T})$ and its derivatives of order $N$ and less, on the hypotenuse $\Gamma_{1}=E_{3}$ and on the $x$ and $y$ axes $\Gamma_{2}=E_{1} \cup E_{2}$. These projectors involve suitable combinations of the Taylor projectors which interpolate $F$ and its derivatives on the sides of the triangle $T$ along parallels to the $x$ and $y$ axes. Explicitly the Taylor projectors are defined by

$$
\begin{aligned}
T_{x}^{2} F & =\sum_{i<N} x^{(i)} F_{i, 0}(0, y), \\
T_{x}^{3} F & =\sum_{i \leqslant N}(x+y-1)^{(i)} F_{i, 0}(1-y, y), \\
T_{y}^{1} F & =\sum_{j \leqslant N} y^{(j)} F_{0, j}(x, 0), \\
T_{y}^{3} F & =\sum_{j \leqslant N}(x+y-1)^{(j)} F_{0, j}(x, 1-x),
\end{aligned}
$$

where $x^{(i)}=x^{i} / i$ ! and $T_{x}^{2}$ denotes the Taylor projector across the side $E_{2}$ along the line through $(x, y)$ parallel to the $x$ axis etc.

Let

$$
\begin{aligned}
P_{2} F= & \left(T_{x}^{2} \oplus T_{y}^{1}\right) F=\sum_{i \leqslant N} x^{(i)} F_{i, 0}(0, y)+\sum_{j \leqslant N} y^{(j)} F_{0, j}(x, 0) \\
& -\sum_{i, j \leqslant N} x^{(i)} y^{(j)}\left(\frac{\partial^{i+j} F}{\partial x^{i} \partial y^{j}}\right)(0,0) .
\end{aligned}
$$

Then it is easily shown that for $F \in C^{N}(\bar{T})$ the conditions of Theorem 2.2 are satisfied 
for the Boolean sum of the projectors $T_{x}^{2}$ on $E_{2}$ and $T_{y}^{1}$ on $E_{1}$ if

$$
\left(\frac{\partial^{m+n} F}{\partial x^{m} \partial y^{n}}\right)(0,0)=\left(\frac{\partial^{n+m} F}{\partial y^{n} \partial x^{m}}\right)(0,0), \quad m, n \leqslant N ; m+n>N
$$

(in which case the Taylor projectors are commutative). Thus for $F$ satisfying the compatibility condition (3.3), $P_{2} F$ interpolates $F$ and its derivatives of order $N$ and less on $\Gamma_{2}=E_{1} \cup E_{2}$. The precision set of $P_{2}$ is the union of those of the two Taylor projectors $T_{x}^{2}$ and $T_{y}^{1}$, namely

$$
x^{i} y^{j}, \begin{cases}0 \leqslant i \leqslant N & \text { for all } j \\ 0 \leqslant j \leqslant N & \text { for all } i\end{cases}
$$

A projector $P_{1}$ is required which interpolates $F$ and its derivatives on $\Gamma_{1}=E_{3}$ and which satisfies the conditions of Theorem 2.2 , namely that $D^{\alpha} P_{1} F$ on $\Gamma_{2}$ is a linear combination of function and derivative evaluations on $\Gamma_{2}$ which are interpolated by $P_{2} F$. This is accomplished by taking a suitable combination of the two hypotenuse Taylor projectors.

Linear Case. (Nielson's interpolant.) Let

$$
P_{1} F=y F(x, 1-x)+x F(1-y, y),
$$

then $P_{1} F$ interpolates $F$ on $\Gamma_{1}=\{x+y=1\}$. Also, on $x=0, P_{1} F=y F(0,1)$ and, on $y=0, P_{1} F=x F(1,0)$. Thus $P_{1} F$ on $\Gamma_{2}$ is a linear combination of function evaluations on $\Gamma_{2}$, and these are interpolated by

$$
P_{2} F=F(0, y)+F(x, 0)-F(0,0) \text {. }
$$

The conditions of Theorem 2.1 are thus satisfied and

$$
\begin{aligned}
\left(P_{1} \oplus P_{2}\right) F= & y F(x, 1-x)+x F(1-y, y)+F(x, 0)+F(0, y)-F(0,0) \\
& -y\{F(0,1-x)+F(x, 0)-F(0,0)\} \\
& -x\{F(0, y)+F(1-y, 0)-F(0,0)\}
\end{aligned}
$$

interpolates $F$ on the boundary $\partial T$ of the triangle $T$. This is a Boolean sum derivation of Nielson's polynomial interpolant.

If we let

$$
\widetilde{F}(x, 0)=(1-x) F(0,0)+x F(1,0), \quad \widetilde{F}(0, y)=(1-y) F(0,0)+y F(0,1),
$$

and $\widetilde{F}(x, 1-x)=F(x, 1-x)$, then

$$
\left(P_{1} \oplus P_{2}\right) \widetilde{F}=y F(x, 1-x)+x F(1-y, y)+(1-x-y) F(0,0)
$$

is an interpolation function which is linear on two sides of the triangle, whilst matching the function $F$ on the other side. This interpolant could be incorporated with piecewise linear finite elements on a triangulated polygon so as to satisfy given boundary conditions exactly.

General Case. Let 


$$
\begin{gathered}
\sum_{i, j \leqslant N} \alpha_{i, j}(x, y)\left(\frac{\partial^{i}}{\partial y^{i}}\left[\frac{\partial}{\partial x}-\frac{\partial}{\partial y}\right]^{j} F\right)(0,1) \\
+\sum_{i, j \leqslant N} \beta_{i, j}(x, y)\left(\frac{\partial^{i}}{\partial x^{i}}\left[\frac{\partial}{\partial x}-\frac{\partial}{\partial y}\right]^{j} F\right)(1,0) \\
\quad+\sum_{i, j \leqslant N} \gamma_{i, j}(x, y)\left(\frac{\partial^{i+j} F}{\partial x^{i} \partial y^{j}}\right)(0,0)
\end{gathered}
$$

where $\alpha_{i, j}, \beta_{i, j}$, and $\gamma_{i, j}$ are the appropriate cardinal functions, be the polynomial interpolant over the $3(N+1)^{2}$ dimensional set of polynomials which are of degree $2 N+1$ along parallels to the three sides of $T$. The case $N=1$ is the tricubic polynomial interpolant of Birkhoff [3] and, for general $N$, the existence of this interpolant is implied by Lemma 4.1 of Barnhill and Mansfield [2]. Then $\alpha_{0,0}(x, y)+\beta_{0,0}(x, y)+$ $\gamma_{0,0}(x, y)=1$ and

$$
\left(D^{\alpha} \alpha_{0,0}\right)\left(E_{1}\right)=\left(D^{\alpha} \beta_{0,0}\right)\left(E_{2}\right)=\left(D^{\alpha} \gamma_{0,0}\right)\left(E_{3}\right)=0 \text { for all }|\alpha| \leqslant N,
$$

where $\left(D^{\alpha} \alpha_{0,0}\right)\left(E_{1}\right)$ represents $D^{\alpha} \alpha_{0,0}(x, y)$ evaluated on the side $E_{1}$ etc. Hence

$$
\left(\alpha_{0,0}+\beta_{0,0}\right)\left(E_{3}\right)=1
$$

and

$$
\left(D^{\alpha}\left[\alpha_{0,0}+\beta_{0,0}\right]\right)\left(E_{3}\right)=0, \quad 1 \leqslant|\alpha| \leqslant N .
$$

Thus

$$
\begin{aligned}
P_{1} F= & \alpha_{0,0}(x, y) T_{y}^{3} F+\beta_{0,0}(x, y) T_{x}^{3} F \\
= & \alpha_{0,0}(x, y) \sum_{j \leqslant N}(x+y-1)^{(j)} F_{0, j}(x, 1-x) \\
& +\beta_{0,0}(x, y) \sum_{i \leqslant N}(x+y-1)^{(i)} F_{i, 0}(1-y, y)
\end{aligned}
$$

is a projector which interpolates $F \in C^{N}(\bar{T})$ and its derivatives of order $N$ and less on $\Gamma_{1}=E_{3}$. Also, for all $|\alpha| \leqslant N, D^{\alpha} P_{1} F$ on $y=0$ involves the derivatives,

$$
\left(\left[-\frac{\partial}{\partial x}+\frac{\partial}{\partial y}\right]^{n} \frac{\partial^{m} F}{\partial x^{m}}\right)(1,0), \quad 0 \leqslant m, n \leqslant N .
$$

$P_{2} F$ defined by Eq. (3.2) interpolates these values provided that $F \in C^{N}(\bar{T})$ satisfies the compatibility condition,

$$
\left(\left[-\frac{\partial}{\partial x}+\frac{\partial}{\partial y}\right]^{n} \frac{\partial^{m} F}{\partial x^{m}}\right)(1,0)=\left(\frac{\partial^{m}}{\partial x^{m}}\left[-\frac{\partial}{\partial x}+\frac{\partial}{\partial y}\right]^{n} F\right)(1,0)
$$

$$
m, n \leqslant N ; m+n>N .
$$

Similarly on $x=0$ we require that 


$$
\begin{aligned}
\left(\left[\frac{\partial}{\partial x}-\frac{\partial}{\partial y}\right]^{m} \frac{\partial^{n} F}{\partial y^{n}}\right)(0,1)=\left(\frac{\partial^{n}}{\partial y^{n}}\left[\frac{\partial}{\partial x}-\frac{\partial}{\partial y}\right]^{m} F\right)(0,1) & \\
& m, n \leqslant N ; m+n>N
\end{aligned}
$$

The conditions of Theorem 2.2 are then satisfied and with (3.3) we have:

THEOREM 3.1. Let $F \in C^{N}(\bar{T})$ and satisfy the compatibility conditions,

$$
\left(\frac{\partial^{m+n} F}{\partial s_{i}^{m} \partial s_{j}^{n}}\right)\left(V_{k}\right)=\left(\frac{\partial^{n+m} F}{\partial s_{j}^{n} \partial s_{i}^{m}}\right)\left(V_{k}\right), \quad m, n \leqslant N ; m+n>N,
$$

at each vertex $V_{k}$ with adjacent sides $E_{i}$ and $E_{j}$, where $\partial / \partial s_{l}$ denotes differentiation along the side $E_{l}$. Then the polynomial Boolean sum function, $\left(P_{1} \oplus P_{2}\right) F=$ $\left(P_{1}+P_{2}-P_{1} P_{2}\right) F$, where $P_{1}$ is defined by (3.12) and $P_{2}$ is defined by (3.2), interpolates $F$ and its derivatives of order $N$ and less on the boundary $\partial T$ of the triangle $T$.

The precision set of the interpolant is that of the projector $P_{2}$; see (3.4).

Examples. (i) For $N=0$,

$$
\alpha_{0,0}(x, y)=y \text { and } \beta_{0,0}(x, y)=x,
$$

giving the linear case (3.5).

(ii) For $N=1$,

$$
\begin{aligned}
& \alpha_{0,0}(x, y)=y^{2}[3-2 y+6 x(1-x-y)] \text { and } \\
& \beta_{0,0}(x, y)=x^{2}[3-2 x+6 y(1-x-y)] .
\end{aligned}
$$

This case is discussed further in Section 4.

4. Removal of Compatibility Conditions. The compatibility conditions (3.15) of Theorem 3.1 can be removed by adding suitable rational terms to the Boolean sum interpolant $\left(P_{1} \oplus P_{2}\right) F$. We consider the rational Hermite projectors on the standard triangle $T$ defined by Eqs. (2.2)-(2.4).

Firstly, since $T_{3}$ interpolates $F$ on $E_{1} \cup E_{2}$, the projector $P_{2}$, defined by (3.2), can be modified to

$$
\widetilde{P}_{2} F=P_{2} F+T_{3}\left(F-P_{2} F\right)
$$

where $T_{3}\left(F-P_{2} F\right)$ is a rational compatibility correction term. We consider now the modified Boolean sum interpolant,

$$
\left(P_{1} \oplus \widetilde{P}_{2}\right) F=\left(P_{1} \oplus P_{2}\right) F+\left(I-P_{1}\right) T_{3}\left(F-P_{2} F\right)
$$

where $P_{1}$ is defined by (3.12). This interpolant requires the compatibility conditions (3.15) at the vertices $V_{1}=(0,1)$ and $V_{2}=(1,0)$. Then $F-\left(P_{1} \oplus \widetilde{P}_{2}\right) F$ has compatible derivatives at the vertex $V_{3}=(0,0)$ and can thus be interpolated by either of the rational Boolean sum operators $T_{1} \oplus T_{2}$ or $T_{2} \oplus T_{1}$. Thus

$$
\left(P_{1} \oplus \widetilde{P}_{2}\right) F+\left(T_{1} \oplus T_{2}\right)\left[F-\left(P_{1} \oplus \widetilde{P}_{2}\right) F\right]
$$

interpolates $F \in C^{N}(\bar{T})$ and its derivatives of order $N$ and less on $\partial T$, where $\left(T_{1} \oplus T_{2}\right)$ - $\left[F-\left(P_{1} \oplus \widetilde{P}_{2}\right) F\right]$ is another rational compatibility correction term. The rational 
terms are zero if the compatibility conditions (3.15) hold.

Example. For $N=1$, the average of (3.2) with the dual expression for $\left(T_{y}^{1} \oplus T_{x}^{2}\right) F$ gives the symmetric projector,

Then

$$
P_{2} F=F(0, y)+x F_{1,0}(0, y)+F(x, 0)+y F_{0,1}(x, 0)
$$

$$
T_{3}\left(F-P_{2} F\right)=\frac{x y(x-y)}{2(x+y)}\left\{\left(\frac{\partial^{2} F}{\partial x \partial y}\right)(0,0)-\left(\frac{\partial^{2} F}{\partial y \partial x}\right)(0,0)\right\}
$$

and the projector

$$
\widetilde{P}_{2} F=P_{2} F+T_{3}\left(F-P_{2} F\right)
$$

interpolates $F \in C^{N}(\bar{T})$ on $\Gamma_{2}=E_{1} \cup E_{2}$. Now

$$
\begin{aligned}
P_{1} F= & y^{2}[3-2 y+6 x(1-x-y)]\left[F(x, 1-x)+(x+y-1) F_{0,1}(x, 1-x)\right] \\
& +x^{2}[3-2 x+6 y(1-x-y)\}\left[F(1-y, y)+(x+y-1) F_{1,0}(1-y, y)\right] .
\end{aligned}
$$

and the Boolean sum $\left(P_{1} \oplus \widetilde{P}_{2}\right) F=\left(P_{1}+\widetilde{P}_{2}-P_{1} \widetilde{P}_{2}\right) F$ can be determined from Eqs. (4.3)-(4.6) where

$$
\begin{aligned}
P_{1} \widetilde{P}_{2} F=y^{2} & {[3-2 y+6 x(1-x-y)] } \\
& \cdot\left[\left(\widetilde{P}_{2} F\right)(x, 1-x)+(x+y-1)\left(\frac{\partial \widetilde{P}_{2} F}{\partial y}\right)(x, 1-x)\right] \\
+ & x^{2}[3-2 x+6 y(1-x-y)] \\
& \cdot\left[\left(\widetilde{P}_{2} F\right)(1-y, y)+(x+y-1)\left(\frac{\partial \widetilde{P}_{2} F}{\partial x}\right)(1-y, y)\right] .
\end{aligned}
$$

It can then be shown that

$$
\left(T_{1} \oplus T_{2}\right)\left[F-\left(P_{1} \oplus \widetilde{P}_{2}\right) F\right]
$$

$$
\begin{array}{r}
=\frac{(x+y-1)^{2} x^{2} y(3-2 x)}{x-1}\left\{\left(\left[\frac{\partial}{\partial x}-\frac{\partial}{\partial y}\right] \frac{\partial F}{\partial x}\right)(1,0)\right. \\
\left.-\left(\frac{\partial}{\partial x}\left[\frac{\partial}{\partial x}-\frac{\partial}{\partial y}\right] F\right)(1,0)\right\} \\
+\frac{(x+y-1)^{2} x y^{2}(3-2 y)}{y-1}\left\{\left(\left[-\frac{\partial}{\partial x}+\frac{\partial}{\partial y}\right] \frac{\partial F}{\partial y}\right)(0,1)\right. \\
\left.-\left(\frac{\partial}{\partial y}\left[-\frac{\partial}{\partial x}+\frac{\partial}{\partial y}\right] F\right)(0,1)\right\}
\end{array}
$$

giving the compatibly corrected interpolant, $\left(P_{1} \oplus \widetilde{P}_{2}\right) F+\left(T_{1} \oplus T_{2}\right)\left[F-\left(P_{1} \oplus \widetilde{P}_{2}\right) F\right]$. 
5. Triangle With One Curved Side. By affine transformation, it is sufficient to consider the triangle with vertices at $V_{1}=(0,1), V_{2}=(1,0)$ and $V_{3}=(0,0)$ and two straight edges along the coordinate axes. We assume that the third side $E_{3}$ opposite the vertex $V_{3}$ is defined by the one-to-one functions,

$$
y=f(x) \text { and } x=g(y),
$$

where $g$ is the inverse function of $f$. The Taylor projectors on $E_{3}$ are now

$$
\begin{aligned}
& T_{x}^{3} F=\sum_{i \leqslant N}[x-g(y)]^{(i)} F_{i, 0}(g(y), y), \\
& T_{y}^{3} F=\sum_{j \leqslant N}[y-f(x)]^{(j)} F_{0, j}(x, f(x)) .
\end{aligned}
$$

The cardinal functions $\alpha_{0,0}(x, y)$ and $\beta_{0,0}(x, y)$ of Section 3 have the properties,

$$
\left[\alpha_{0,0}(1-f(x), y)+\beta_{0,0}(1-f(x), y)\right]\left(E_{3}\right)=1
$$

and

$$
\left[D^{\alpha} \alpha_{0,0}(1-f(x), y)+D^{\alpha} \beta_{0,0}(1-f(x), y)\right]\left(E_{3}\right)=0,
$$

for $1 \leqslant|\alpha| \leqslant N$. Thus

$$
P_{1} F=\alpha_{0,0}(1-f(x), y) T_{y}^{3} F+\beta_{0,0}(1-f(x), y) T_{x}^{3} F
$$

is a suitable projector on $E_{3}$. The dual projector is

$$
P_{1} F=\alpha_{0,0}(x, 1-g(y)) T_{y}^{3} F+\beta_{0,0}(x, 1-g(y)) T_{x}^{3} F
$$

or alternatively an average of these two can be considered.

The Boolean sum function $\left(P_{1} \oplus P_{2}\right) F$, where $P_{2}$ is defined by (3.2), gives a blending function interpolant on the curved triangle.

Examples. For the case $N=0,(5.1),(5.2),(5.3)$ and (3.16) give the projector,

$$
P_{1} F=y F(x, f(x))+[1-f(x)] F(g(y), y) .
$$

From (3.2)

$$
P_{2} F=F(0, y)+F(x, 0)-F(0,0),
$$

so that

$$
\begin{aligned}
\left(P_{1} \oplus P_{2}\right) F= & y F(x, f(x))+[1-f(x)] F(g(y), y)+F(0, y)+F(x, 0) \\
& -F(0,0)-y[F(0, f(x))+F(x, 0)-F(0,0)] \\
& -[1-f(x)][F(0, y)+F(g(y), 0)-F(0,0)] .
\end{aligned}
$$

For the case $N=1,(5.1)-(5.3)$ and (3.17) give the projector,

where

$$
\begin{aligned}
P_{1} F=\alpha_{0,0} & (1-f(x), y)\left\{F(x, f(x))+[y-f(x)] F_{0,1}(x, f(x))\right\} \\
& +\beta_{0,0}(1-f(x), y)\left\{F(g(y), y)+[x-g(y)] F_{1,0}(g(y), y)\right\},
\end{aligned}
$$




$$
\begin{aligned}
& \alpha_{0,0}(1-f(x), y)=y^{2}[3-8 y+6 f(x)\{1+y-f(x)\}] \text { and } \\
& \beta_{0,0}(1-f(x), y)=[1-f(x)]^{2}[1+2 f(x)+6 y\{f(x)-y\}] .
\end{aligned}
$$

From (3.2),

$$
\begin{aligned}
P_{2} F= & F(0, y)+x F_{1,0}(0, y)+F(x, 0)+y F_{0,1}(x, 0) \\
& -\left\{F(0,0)+y F_{0,1}(0,0)+x F_{1,0}(0,0)+x y F_{1,1}(0,0)\right\}
\end{aligned}
$$

Hence

$$
\begin{gathered}
P_{1} P_{2} F=\alpha_{0,0}(1-f(x), y)\left\{F(0, f(x))+x F_{1,0}(0, f(x))+F(x, 0)+f(x) F_{0,1}(x, 0)\right. \\
-\left[F(0,0)+f(x) F_{0,1}(0,0)+x F_{1,0}(0,0)\right. \\
\left.+x f(x) F_{1,1}(0,0)\right] \\
+[y-f(x)]\left[F_{0,1}(0, f(x))+x F_{1,1}(0, f(x))\right. \\
\left.\left.+F_{0,1}(x, 0)-F_{0,1}(0,0)-x F_{1,1}(0,0)\right]\right\} \\
(5.11) \quad+\quad+F_{0,0}(1-f(x), y)\left\{F(0, y)+g(y) F_{1,0}(0, y)+F(y), 0\right)+y F_{0,1}(g(y), 0) \\
-\left[F(0,0)+y F_{0,1}(0,0)+g(y) F_{1,0}(0,0)\right. \\
\left.+y g(y) F_{1,1}(0,0)\right] \\
+[x-g(y)]\left[F_{1,0}(0, y)+F_{1,0}(g(y), 0) \quad\right. \\
\left.\left.+y F_{1,1}(g(y), 0)-F_{1,0}(0,0)-y F_{1,1}(0,0)\right]\right\} .
\end{gathered}
$$

Equations (5.8)-(5.11) completely define the Boolean sum interpolant $\left(P_{1} \oplus P_{2}\right) F=$ $\left(P_{1}+P_{2}-P_{1} P_{2}\right) F$.

Mathematics Department

University of Utah

Salt Lake City, Utah 84112

Mathematics Department

Brunel University

Uxbridge, Middlesex, England

1. R. E. BARNHILL, G. BIRKHOFF \& W. J. GORDON, "Smooth interpolation in triangles," J. Approximation Theory, v. 8, 1973, pp. 114-128.

2. R. E. BARNHILL \& L. MANSFIELD, "Error bounds for smooth interpolation in triangles," J. Approximation Theory, v. 11, 1974, pp. 306-318.

3. G. BIRKHOFF, “Tricubic polynomial interpolation,” Proc. Nat. Acad. Sci. U.S.A, v. 68, 1971, pp. 1162-1164. MR 45 \#9030.

4. W. J. GORDON \& J. A. WIXOM, "Pseudo-harmonic interpolation on convex domains," SIAM J. Numer. Anal., v. 11, 1974, pp. 909-933.

5. J. A. MARSHALL \& A. R. MITCHELL, "An exact boundary technique for improved accuracy in the finite element method," J. Inst. Math. Appl., v. 12, 1973, pp. 355-362.

6. G. M. NIELSON, Private communication, Baltimore, June 1972. 